\title{
Countermeasures for Hemodialysis Patients with Fever in Non-Designated Hospitals during the Epidemic Period of Novel Coronavirus Pneumonia (COVID-19)
}

\author{
Yu Chen, Jinglan Luo*, Yingxue Zhong, Zongchao Yu, Lianghong Yin, Yunyi Li, Ling Liu, \\ Wenxue Liang\#, Fanna Liu\# \\ Nephrology Department, The First Affiliated Hospital of Jinan University, Guangzhou, China \\ Email: 1139795771@qq.com, "1005966978@qq.com," 81968920@qq.com
}

How to cite this paper: Chen, Y., Luo, J.L., Zhong, Y.X., Yu, Z.C., Yin, L.H., Li, Y.Y., Liu, L., Liang, W.X. and Liu, F.N. (2020) Countermeasures for Hemodialysis Patients with Fever in Non-Designated Hospitals during the Epidemic Period of Novel Coronavirus Pneumonia (COVID-19). Open Journal of Epidemiology, 10, 179-186. https://doi.org/10.4236/ojepi.2020.102016

Received: April 13, 2020

Accepted: May 10, 2020

Published: May 13, 2020

Copyright $\odot 2020$ by author(s) and Scientific Research Publishing Inc. This work is licensed under the Creative Commons Attribution International License (CC BY 4.0).

http://creativecommons.org/licenses/by/4.0/

\begin{abstract}
Objective: To explore the effect of countermeasures for hemodialysis patients with fever during the incubation period of novel coronavirus pneumonia (NCP) in a non-designated hospital. Methods: For 22 hemodialysis patients with fever in our hospital, we developed a specialist program based on the latest NCP treatment guidelines for nephrology and hemodialysis. We trained medical staff accurately and rapidly online. According to the specialist program, we implemented meticulous treatment for patients including dialysis, psychological and environmental care. We provided protection of medical staff in wards and dialysis rooms. Medical waste was deposited properly. Moreover, we implemented quality improvement in a timely manner according to the situation. Results: The 22 hemodialysis patients with fever in our hospital were not infected with novel coronavirus 2019. The epidemiological screening rate was $100 \%$, and the percentage of family care giving was reduced by $29.8 \%$. All medical stuff were uninfected. The patient's satisfaction rate in the observation group is higher than that in the control group ( $96 \%$ vs. $74 \%, \mathrm{p}=0.048)$. Conclusion: The countermeasures for hemodialysis patients with fever developed by our specialty were effective. We can satisfy requirements for the NCP prevention for hemodialysis patients with fever in non-designated hospitals through implementing these countermeasures.
\end{abstract}

\section{Keywords}

Novel Coronavirus Pneumonia, Countermeasures, Fever, Hemodialysis 


\section{Introduction}

On December 31, 2019, a case of pneumonia of unknown cause was reported in Wuhan, Hubei Province, China. On January 9, 2020, the Chinese Center for Disease Control and Prevention reported that the disease was caused by a novel coronavirus (2019-nCoV) that belongs to the severe acute respiratory syndrome (SARS) CoV family [1]. WHO named it 2019-nCoV on January 12, 2020. Current epidemiological data indicate that person-to-person spread of 2019-nCoV has been occurring [1] [2]. As of 22:00 hours on February 22, 2020,online public data from the National Health Commission, the Provincial and Municipal Health Commissions, the official channels of Hong Kong, Macao and Taiwan, showed that there were 76,395 cases of NCP confirmed, 5365 cases suspected, and 21,068 cases cured in China. The situation is still serious.

There are 140 million people in China suffering from chronic kidney disease. By the end of 2018, the National Dialysis Registration System showed that there were $>670,000$ patients on maintenance dialysis. Hemodialysis patients are also susceptible to NCP. It is especially important to prevent hemodialysis patients with fever from developing NCP infection. As one of the non-designated hospitals for NCP patients, 22 patients with febrile dialysis were admitted to our hospital from January 23 to March 23, 2020. They were further nursed with a specialist program developed by the Department of Nephrology in our hospital.

\section{Method}

\subsection{Information about Characteristics of Patients}

From January 23, 2020 to March 23, 2020, the number of admitted patients to the nephrology ward in our hospital was 174, 96 males and 78 females, including 22 hemodialysis patients with fever (12 males and 10 females; average age 63.77 years). All dialysis patients suffered from chronic kidney diseases stage 5 (CKD5). The causes of fever were: Vascular access related infection, pulmonary infection, urinary tract infection, upper respiratory tract infection (URTI), and other reason (see Table 1 ). Patients in observation group $(n=22)$ were chose according to inclusion criteria: hemodialysis patients admitted to the nephrology ward from January 23, 2020 to March 23, 2020 and the body temperature measured under armpit was higher then $37.3^{\circ} \mathrm{C}$. Patients in control group $(n=59)$ were chose according the same criteria during January 23 to March 232019.

\subsection{Nursing Countermeasures}

\subsubsection{Develop a New Program and Provide Accurate and Rapid Training to All Medical Staffs}

According to both the latest NCP guidance and the measures in our hospital, we developed countermeasures for patients in the Nephrology ward and the Hemodialysis Center in our hospital. We provided training to all medical workers accurately and rapidly online. When necessary, psychological counseling was provided to relieve stress to ensure the medical staff went on duty safely. 
Table 1. Characters of two groups (n).

\begin{tabular}{cccc}
\hline characters & $\begin{array}{c}\text { Observation group } \\
(\mathrm{N}=22)\end{array}$ & $\begin{array}{c}\text { Control group } \\
(\mathrm{N}=59)\end{array}$ & p-value \\
\hline Age (years) & 63.77 & 64 & 0.95 \\
Sex (male) (\%) & $13(59.1 \%)$ & $28(47.5 \%)$ & 0.50 \\
Epidemiological contact history & 1 & 0 & 0.27 \\
cough & 46 & 15 & 0.54 \\
Reasons for fever & & & \\
Vascular access related infection & 1 & 3 & 1.00 \\
Pulmonary infection & 15 & 22 & 0.025 \\
Urinary tract infection & 0 & 1 & 1 \\
Upper respiratory tract infection (URTI) & 11 & 0 & 0.03 \\
Other reason for fever & 6 & 20 & 0.76 \\
\hline
\end{tabular}

\subsubsection{Countermeasures in the Ward}

\section{1) Implementation of the new specialty program in the ward to ensure safety}

a) Before admission to the hospital, adult patients are requested to undergo lung computed tomography (CT) and pediatric patients to undergo chest X-ray instead of chest CT. Nucleic acid testing, routine blood examination, and $\mathrm{C}$-reactive protein and influenza $\mathrm{A} / \mathrm{B}$ antigen testing in combination with the $\mathrm{CT}$ results are required before admission, and these tests are checked by the admission office.

b) After admission, the patients are sent to a special isolation ward equipped with water dispensers and other necessities of life. If NCP infection is excluded, the patients are transferred to a general ward; if NCP in fection is suspected or confirmed, it must be reported immediately to the Hospital-Acquired Infection Control Department as well as the Department of Preventive Medicine in the hospital, and the patient should be transferred to one of the designated hospitals for treatment.

c) Timely communication with the Logistics Department is ensured so that after elevators are used by the patients, they are sterilized immediately. If the patient use the stairs, the stairs and the surrounding surfaces, including the ceiling, are sprayed with $1000 \mathrm{mg} / \mathrm{L}$ chlorine-containing disinfectants, and then the key contaminated parts, such as elevator buttons and the floor, are further wiped and disinfected with $1 \mathrm{~g} / \mathrm{L}$ chlorine-containing disinfectants, and then lastly with clean water [3].

d) The air in the ward is naturally ventilated to keep air flowing, while the ward doors always remain closed. The surface of medical facilities, equipment, bed rails, bedside tables, and other objects in the ward should be wiped and disinfected at least twice a day with $1 \mathrm{~g} / \mathrm{L}$ chlorine-containing disinfectants. They are disinfected at any time if contaminated. The floor of the ward should be wiped and disinfected at least twice a day with $1 \mathrm{~g} / \mathrm{L}$ chlorine-containing disin- 
fectant. If NCP or suspected NCP is excluded, the ward that the hemodialysis patients with fever have left is disinfected in the same manner as the general ward [4].

2) Protection of medical personnel and observation of patients' status

a) An access control system is strictly implemented: entry of unrelated personnel is strictly limited, and those with a body temperature $>37.3^{\circ} \mathrm{C}$ are prohibited. A reminder stating the requirements for epidemic prevention is posted at the ward door. Escorts are required to fill out the Registration Form for NCP Screening of the First Affiliated Hospital of the University. Accompanying cards are issued to the escorts according to their ID. The number of escorts is counted daily and reported to the hospital. Moreover, a non-contact thermometer is used to measure body temperature and the results registered. Patients and escorts are instructed on how to ware face masks correctly.

b) The patients follow the doctors' advice on the use of medication. The treatment time is controlled and fixed to a certain period of time daily. To reduce the risk of infection and the use of protective materials, the number of times that medical staffs enter and leave the ward is reduced.

c) During patient visits, medical staff are required to take protective measures during treatment, including strict implementation of the 7-steps hand-washing method, wearing N95 medical masks, goggles, gowns, hats, shoe covers and gloves. When the patients have fever, medical staff need to follow doctors' advice to give the patient antipyretic drugs or ice packs to cool down. When patients' body temperature is $\geq 38.5^{\circ} \mathrm{C}$, blood samples are withdrawn for examination according to the doctors' instructions, and the temperature changes are reported to the doctors after treatment. Daily fever is recorded and reported.

\section{3) Proper handling of medical waste}

a) Clothing treatment. All clothing should be classified as infectious fabric. After disposal of the infectious fabric in the yellow medical waste bag, which is designated for infectious fabric, proper labeling should be posted, and the source information written down. The collected medical waste bags should be handed over to the cleaning and clothing department, where the infectious fabric should be disinfected and sterilized before cleaning.

b) Disposal of cleaning tools. Disposable rags are soaked in $2 \mathrm{~g} / \mathrm{L}$ chlorine-containing disinfectant and further used to disinfect the surface of medical vehicles and other items. Disposable rags are treated as medical waste after usage. Towels are soaked with $2 \mathrm{~g} / \mathrm{L}$ chlorine-containing disinfectant for $30 \mathrm{mi}$ nutes. The staff living area and the contaminated area of the soaking bucket must be separated and clearly marked. Mops used to disinfect the floor should be soaked in $2 \mathrm{~g} / \mathrm{L}$ chlorine disinfectant for 30 minutes after usage [4], and these are recorded daily.

\subsubsection{Countermeasures in the Hemodialysis Center}

\section{1) Environmental and material preparation}

a) Doctors can take advantage of the network to strengthen their communica- 
tions with the patients. A special communication group can be established for dialysis patients. Patients or family members can scan the QR code to enter the group. Issues and notifications are posted and discussed in the group. However, escorts are not allowed to join the group. Reminders are posted at the center entrance. The dialysis center's television broadcasts information about NCP prevention and control measures.

b) The dialysis center maintains air circulation and adopts natural ventilation. The door of each dialysis room is always kept closed and the windows are open. Wipe disinfection of the ground, diagnosis and treatment facilities, equipment surfaces, bed rails, bedside tables, and other surfaces is performed at least twice a day with a disinfectant containing $100 \mathrm{mg} / \mathrm{L}$ chlorine.

c) The dialysis shift for febrile patients should be the last hemodialysis shift of the day, which is different from that of other patients. It is necessary to place the patients in an separate dialysis treatment room that is not located in areas with positive blood-borne diseases. The treatment room should be disinfected after use.

d) The dialysis treatment room should be disinfected by chlorine-containing disinfectant spray or ultraviolet irradiation for more than 1 hour after each treatment. Disinfection records should be monitored daily. If the surfaces and floors of machines, beds, and other objects are contaminated by patients' blood and feces, the visible contamination is firstly removed by absorbent materials such as paper towels, and further disinfected using $2 \mathrm{~g} / \mathrm{L}$ chlorine-containing disinfectant. These procedures are recorded every time [5].

2) Strengthen personnel training and arrange shifts reasonably to ensure normal operation of the work

All staff are trained in NCP infection control and specialized countermeasures developed in our hospital. Medical staff should take protective measures during dialysis treatment, strictly following the seven-step hand washing method, wearing N95 medical masks, goggles, face shield, isolation clothing, hats, shoe covers and gloves [6].

3) Correct implementation of doctors' instructions and close observation of patients' status

a) Body weight is measured before and after treatment to set the ultrafiltration amount according to the dry body weight. The dialysis mode is implemented in accordance with the doctors' advice. The dialyzer and tubing are completely sealed during dialysis. The dialysis blood flow is $200-250 \mathrm{ml} / \mathrm{min}$. The ultrafiltration rate is adjusted according to the patients' blood pressure, so as not to cause complications, such as hypotension caused by rapid ultrafiltration [7]. During the whole treatment period, electrocardiography is monitored, and the patients' vital signs are closely observed. Blood pressure is measured once every hour. For those with unstable vital signs, blood pressure should be measured every 15 - 30 minutes, and any changes in respiratory rate and blood oxygen saturation noted. Various dialysis parameters should be adjusted in time to ensure successful completion of treatment. 
b) The latency of 2019-nCoV is $1-14$ days, usually 3 - 7 days [8]. Thus, after 14 days of dialysis in a single separate room, patients without any infection symptoms can enter the normal dialysis area.

4) Medical waste should be disposed properly to prevent cross-infection

The clothing and cleaning tools are handled in the same way as in the Nephrology Ward.

\subsection{Observation and Follow up}

We developed a specialist nephrology program to treat and nurse them during this specific time. All of patients ware followed up during their hospitalization. At the same time, we closely observed NCP infection and screening rates, average Hospitalization days, as well as the percentage of family care giving (Family care givers/patients $\times 100 \%)$.

\subsection{Statistical Analysis}

SPSS 22.0 statistical software was used for statistical analysis of the data. The count data was expressed as a percentage. The t-test was used to analyze the numerical data and the count data was analyzed by the chi-square (or fisher) test. The test level was $\alpha=0.05, \mathrm{P}<0.05$ was considered statistically significant.

\section{Results}

From January 23, 2020 to March 23, 2020, a total of 174 patients were admitted to the ward in our Department of Nephrology, including 22 hemodialysis patients with fever. After the specific plans developed by us were implemented, rational treatment and accurate protection were carried out on 22 patients with fever and dialysis. As a result, none of the medical staff were infected with COVID-19. The mean of hospitalization days in the observation group is 19.1 days and that in the control group is 14.4 days, but there is not statistical difference between both groups $(\mathrm{p}=0.07)$. The NCP epidemiological screening rate of inpatients is $100 \%$, and the percentage of family care giving is reduced by $29.8 \%$. The patient's satisfaction rate in the observation group is higher then that in the control group (96\% vs $74 \%, \mathrm{p}=0.048$ ) (see Table 2 ).

\section{Discussion}

The major route of transmission of 2019-nCOV is through respiratory droplets and contact [9]. Due to its strong contagion, the population is generally susceptible, especially the elderly. Hemodialysis patients are inherently less resistant to

Table 2. Results of two groups.

\begin{tabular}{cccc}
\hline & Observation group & Control group & P-value \\
\hline Mean of Hospitalization days & 19.1 & 14.4 & 0.07 \\
Percentage of family care giving & $35.6 \%$ & $65.4 \%$ & \\
Patient's satisfaction rate & $96 \%$ & $74 \%$ & 0.048 \\
\hline
\end{tabular}


infection and they need to travel back and forth between the hospital and home three times a week. This situation creates a higher risk of infection than for healthy individuals. If dialysis patients suffer from NCP infection, they need to be isolated for dialysis treatment, which also brings challenges to the dialysis center. Our hospital has treated 22 patients with febrile dialysis. Based on the original nursing process of the nephrology ward and blood purification Centre, we worked together with the infection control experts in our hospital and published a new specialty nursing process for patients in Blood Purification Center and the nursing process for inpatients in the department of nephrology. Moreover, according to "Expert Recommendations for the Prevention and Control of New Coronavirus Pneumonia in Hemodialysis Units (Centers)" published by National Kidney Disease Medical Quality Control Center and "Guiding Opinions on Medical Work During Coronavirus Infection Prevention and Control from Nephrologist Branch of the Chinese Medical Doctors Association" published on February 1, 2020 [5], we continuously modify and improve the program to provide scientific guidance for nurses. Additionally, we organized multiple trainings for NCP infection and prevention skills on the Internet, which reduced the risk of infection for medical staff and ensured the safety of all parties in the hospital. This countermeasure provides reference for non-specialized hospitals to treat patients with febrile dialysis during NCP.

\section{Conclusion}

Our hospital, which is one of the non-designated hospitals during the NCP outbreak, formulated a special plan for inpatients with hemodialysis fever. By standardized management of the ward environment, management of medical staff, and handling of medical waste, we carefully treated and nursed 22 patients with hemodialysis fever. We monitored the NCP infection, NCP screening and ward care rates, and we found out that the NCP infection rate of 22 patients was 0 , their medical condition was stable, and all the medical staff in contact with the patients were not infected and in good health. The NCP screening rate of patients and their families was $100 \%$, and the rate of hospitalization and escorting decreased by $38 \%$ compared with the same period last year. We can satisfy requirements for the NCP prevention for hemodialysis patients with fever in non-designated hospitals through implementing these countermeasures.

\section{Conflicts of Interest}

The authors declare no conflicts of interest regarding the publication of this paper.

\section{References}

[1] Euro Surveillance Editorial Team (2020) Note from the Editors: World Health Organization Declares Novel Coronavirus (2019-nCoV) Sixth Public Health Emergency of International Concern. https://doi.org/10.2807/1560-7917.ES.2020.25.5.200131e 
[2] Holshue, M.L., DeBolt, C., Lindquist, S., Lofy, K.H., Wiesman, J., Bruce, H., Spitters, C., et al. (2020) First Case of 2019 Novel Coronavirus in the United States. New England Journal of Medicine, 382, 929-936. https://doi.org/10.1056/NEJMoa2001191

[3] Xu, C.-J., Jin, J.-F. and Song, J.-P. (2020) Application of Refined Management in Prevention and Control of Coronavirus Disease 2019 Epidemic in Non-Isolated Areas of a General Hospital. Chinese Journal of Nursing, 55, E004. https://doi.org/10.1016/j.ijnss.2020.04.003

[4] Li, L.-Y., Gong, Y.-X. and Guo, Y.-H. (2012) Background, Significance, Main Contents and Characteristics of Technical Specification for Disinfection of Medical Institutions. Chinese Nursing Management, 12, 7-9.

[5] Ministry of Health of the People's Republic of China (2004) Methods for the Management of Medical Waste in Medical and Health Institutions. China Environmental Protection Industry, 10-13.

[6] National Medical Quality Control Center for Kidney Diseases (2020) Expert Advice for Prevention and Control of Novel Coronavirus Pneumonia in Hemodialysis Room (Center). http://www.cnrds.net/Static/OfficialDocumentDown.html

[7] Miao, X.P. (2019) An Effective Nursing Method for Hypotension in Patients with Uremia during Hemodialysis. World Latest Medicine Information, 19, 217-218.

[8] National Health Committee of the People's Republic of China (2020) Guideline for Diagnosis and Treatment of Novel Coronavirus Pneumonia (Trial Version Sixth) http://www.nhc.gov.cn/xcs/zhengcwj/202002/8334a8326dd94d329df351d7da8aefc2. $\underline{\text { shtml }}$

[9] General Office of the National Health Commission (2020) Covid-19 Prevention and Control Plan (Version Fourth).

http://www.gov.cn/zhengce/zhengceku/2020-02/07/content 5475813.htm 